I doia Fernández, Genaro Guisasola, Mikel Garmendia, Itziar Alkorta \& Alba Madinabeitia (2013) “¿Puede la formación tener efectos globales en la universidad? Desarrollo docente, metodologías acticas y curriculum híbrido." Infancia y Aprendizaje, 36:3, 387-400

"This is an Accepted Manuscript of an article published by Taylor \& Francis Group in Infancia y Aprendizaje: J ournal for the Study of Education and Development on 23/01/2014, available online:

http://www.tandfonline.com/doi/abs/10.1174/ 021037013807532990

http://dx.doi.org/10.1174/021037013807532 $\underline{990}$ 


\title{
¿PUEDE LA FORMACIÓN TENER EFECTOS GLOBALES EN LA UNIVERSIDAD? DESARROLLO DOCENTE, METODOLOGÍAS ACTICAS YCURRICULUM HIBRIDO
}

\begin{abstract}
Palabras clave: Educación Superior, Desarrollo Docente, Formación, Aprendizaje basado en problemas, Desarrollo educacional, Liderazgo educacional, Cambio organizativo.

Partiendo de un concepto comprensivo del desarrollo docente que pretende influir tanto en los niveles individuales (habilidades, concepciones) como en los organizativos e institucionales, analizaremos el programa ERAGIN para desarrollo de metodologías activas (Método de Caso, Aprendizaje basado en Problemas y Aprendizaje basado en Proyectos) en el que han participado ya más de 200 profesores durante los últimos cuatro años. A través de una estrategia larga en el tiempo (350 horas) y trabajando en grupos de co-mentoría, el estudio empírico da evidencias del impacto de esta formación en los conceptos sobre la enseñanza y el aprendizaje (E-A) de los docentes, en su desempeño pero también en la capacidad para investigar la docencia (scholarship of teaching and learning) o para liderar en ámbitos docentes, aspectos todos que nos introducen ya en el cambio organizativo y colaboran en la progresiva materialización del curriculum híbrido.
\end{abstract}

\section{DOES TEACHER TRAINING HAVE GLOBAL EFFECTS IN THE UNIVERSITY? FACULTY DEVELOPMENT, ACTIVE METHODOLOGIES AND HYBRID CURRICULUM}

Keywords: Higher education, Faculty Development, Instructional development, Instructional training, Problem-based learning, Educational development, Educational leadership, Scholarship of teaching and learning, Organizational change.

Based on a comprehensive concept of faculty development that aims to influence both individual (skills, concepts) and the organizational and institutional levels, we will focus on ERAGIN program. This program, in which more than 200 teachers have participated over the past four years, deals with active methodologies (Case Method, Problem-Based Learning and Project-Based Learning), through a long-term strategy (350 hours) and working in co-mentoring groups. Our study provides empirical evidence of the impact not only on teaching skills and conceptions about teaching and learning (T-L) but also on the ability for T-L research (scholarship of teaching and learning) as well as on educational leadership. Those aspects point out organizational change and support the progressive realization of the hybrid curriculum.

\section{Agradecimientos}

Este trabajo se ha llevado a cabo en el marco del Vicerrectorado de Calidad e Innovación de la UPV/EHU y ha sido desarrollado en colaboración por dos Grupos de Investigación. El Grupo de Investigación IkasGura: Cambio educativo en la universidad (GIU 11/14) financiado por la UPV/EHU y el Grupo de Investigación en enseñanza de la física, las matemáticas y la tecnología (GIEFMYT) (GIC 10/87) financiado por el Gobierno Vasco.

Queremos agradecer a los profesores Luis Branda de la Universidad de Girona y Jorge González del Politécnico de Monterrey (México) su colaboración activa en el programa y su valioso asesoramiento.

Fernández, Idoia (1); Guisasola, Genaro (2); Garmendia, Mikel (3); Alkorta, Itziar (4); Madinabeitia, Alba (5).

UPV-EHU. Universidad del País Vasco-Euskal Herriko Unibertsitatea

(1) Facultad de Filosofía y Ciencias de la Educación, Dpto. Teoría e Historia de la Educación. Avda. de Tolosa, 70, 20018 Donostia. Teléfono 943015606. Correo electrónico: idoia.fernandez@ehu.es

(2) Escuela Universitaria Politécnica de Donostia-San Sebastián, Dpto. Física I. Plaza de Europa 1 20018 Donostia. 943018622 jenaro.guisasola@ehu.es 
(3) Escuela Universitaria Politécnica de Donostia-San Sebastián, Dpto. Expresión Gráfica y Proyectos de Ingeniería, Plaza de Europa 1, 20018 Donostia. 94301 8671, mikel.garmendia@ehu.es.

(4) Facultad de Derecho. Dpto. Derecho Civil. Po Manuel de Lardizábal, no 2, 20018 Donostia, 943015240 , itziar.alkorta@ehu.es

(5) Facultad de Filosofía y Ciencias de la Educación, Dpto. Teoría e Historia de la Educación. Avda. de Tolosa, 70, 20018 Donostia. Teléfono 94601 2200. Correo electrónico: alba.madinabeitia@ehu.es

Autora Responsable: Idoia Fernández

\section{Introducción}

Las universidades suelen recurrir a la formación del profesorado como un recurso básico para transitar al "modelo de aprendizaje centrado en el estudiante" es decir, aquel en el que la transmisión del conocimiento disciplinar por parte del profesor da paso a otro en el que éste se convierte en guía de un estudiante para que sea capaz de responder a nuevos problemas (problem solving). Tomando como referencia el análisis de Gibbons, Limoges, Nowotny y Schwartzman (1994), Barnett (2000) señala que la Educación Superior (ES) debe afrontar el reto de hacer convivir dos modos de conocimiento o epistemologías en el curriculum. Por una parte el relativo a amplios corpus disciplinares en los que el conocimiento es proposicional, se publica en las revistas científicas y se somete a revisión y crítica de los pares (Modo 1 de conocimiento). Y, por otro lado, otra forma de conocimiento más práctico, táctil y comunicativo que se produce en tiempo real y consiste en solucionar situaciones y problemas (Modo 2 de conocimiento).

La traducción de esta idea a la ES es diversa: desde curriculos basados en el conocimiento disciplinar (aderezados con el discurso de las competencias) a universidades que han diseñado auténticos curriculos alternativos basados en la resolución de problemas, como es el caso de la emblemática Universidad de MacMaster (Araujo y Sastre, 2008) o el reciente modelo de la Team Academy de Jyväskylä University of Applied Sciences (Finlandia). Entre ambos polos encontramos el curriculum híbrido (Armstrong, 1997) el que conviven el aprendizaje a través de casos, problemas o proyectos junto con la enseñanza disciplinar y que tiene con fin último incrementar el aprendizaje activo o disminuir la experiencias de aprendizaje pasivo que se producen en las clases magistrales. En el caso de la Universidad del País Vasco-Euskal Herriko Unibertsitatea (UPV/EHU) el objetivo institucional es generar progresivamente un curriculum híbrido en el que el aprendizaje del Modo 2 de conocimiento esté presente de forma visible y sustentado en evidencia empírica, ya que trabajamos desde la premisa de que todos nuestros estudiantes adquieren un conocimiento disciplinar suficiente y satisfactorio, pero su capacidad para desenvolverse con naturalidad en la resolución es problemas abiertos es más dudosa. Ahora bien, de acuerdo con Barnett (2000) pensamos que ambos modos de conocimiento son necesarios y complementarios, por lo que ambos deben estar presentes en el curriculum. El programa que presentamos pretende precisamente sentar las bases para hibridar el curriculum al menos en un $10 \%$ del total de créditos impartidos, objetivo estratégico que la universidad se ha propuesto para el año 2017 (UPV/EHU, 2012).

Al hilo de lo anterior, esta investigación analiza un programa para el desarrollo de metodologías activas, en concreto el Método de Caso (MdC), el Aprendizaje basado en Problemas (ABP) y el Aprendizaje basado en Proyectos (ABPy). Existe una amplia bibliografía sobre la formación de tutores/instructores de ABP pero se han producido en universidades en las que el uso de estas metodologías está institucionalizado y pre-establecido, es decir, los problemas, proyectos y casos están diseñados y el empeño de los responsables de la formación es desarrollar estrategias eficaces para que los tutores de ABP apliquen con éxito estas metodologías con los estudiantes (Barrows, 1988; Farmer, 2004; Irby, 1996). Sin embargo hay poca evidencia empírica en entornos donde el intento sea introducir cambios en un curriculum disciplinar pre-existente de manera que los docentes se formen para ser "productores expertos" de problemas, proyectos o casos y que se activen más allá de su aula en aras de producir e institucionalizar nuevos desarrollos organizativos y curriculares.

En este artículo presentaremos el marco teórico sobre el que se basa este programa, el estudio empírico del impacto en los conceptos sobre la enseñanza y el aprendizaje (E-A) de los docentes o en su desempeño pero también en la capacidad para investigar la docencia (scholarship of teaching and learning) o para liderar en ámbitos docentes, aspectos todos que nos introducen ya en el cambio organizativo y colaboran en la progresiva materialización del curriculum híbrido. Finalmente apuntaremos algunas conclusiones y retos tras cuatro años de andadura y más de 200 profesores formados. 


\section{Marco teórico y diseño de un programa de desarrollo docente prolongado en el tiempo y articulado a través de la co-mentoría.}

Los interrogantes que nos planteamos fueron: ¿Qué pasos puede dar una universidad organizada disciplinalmente para introducir de manera efectiva en el curriculum nuevas formas de aprender vinculadas a la resolución de problemas reales? ¿Cómo atraer al profesorado a formarse en metodologías docentes de corte más inductivo (Modo 2 de conocimiento) cuando toda su socialización previa es precisamente deductiva (Modo 1 de conocimiento)? ¿Qué estrategias de desarrollo docente son efectivas para provocar que este cambio se produzca de forma real y profunda?

Para dar respuesta a estos interrogantes, necesitamos revisar qué se entiende por formación y qué nos dice la investigación científica al respecto. La primera dificultad es terminológica. Países como USA, Canadá, UK y Australia empezaron a institucionalizar servicios de formación de su profesorado universitario en la década de los 70 (Gaff y Simpson, 1994; Gibbs, 2004; Gosling, 2008). Proliferaron, así, seminarios, cursos y talleres guiados por la idea de que la formación mejoraría la calidad de la enseñanza. Influenciados por el conductismo dominante en esta época, estas acciones se articulaban como una suerte de ejercicio, entrenamiento (training), una preparación para el desempeño en donde predominantemente se buscaba una mejora en las habilidades (skills) de cada docente en términos de conducta (Wilkerson e Irby, 1998: 388-89). Este enfoque teórico fue mutando en la década de los 80 a enfoques cognitivistas que pusieron su énfasis en el cambio de concepciones fraguando lo que Shulman (1987) denominó Contenido Práctico de la Enseñanza y a orientaciones como la Epistemología de la Práctica que subraya la importancia de la reflexión en y sobre la práctica (Schön, 1992). En los 90 el socio-constructivismo despega el aprendizaje de su esfera individual y lo define como una negociación colectiva de significados, es decir, una actividad que entraña el intercambio, la cooperación y la comunicación. Boyer (1990) irrumpe en este encuadre con otro concepto de difícil traducción, scholarship of teaching (and learning), traducido un tanto forzadamente como "excelencia visible en la enseñanza" (Bolívar, 2008) o "dimensión académica de la enseñanza y el aprendizaje" (Morales, 2010) y de la necesidad de llevarla hasta el ámbito de la producción de evidencia empírica, escrutinio de pares y difusión en revistas especializadas En este marco las comunidades de práctica, los proyectos de I/A o la co-mentoría son estrategias que van adquiriendo importancia.

A medida que se han producido estas mutaciones teóricas, la terminología ha ido cambiando. Si bien el término "formación" (training) se mantiene en la literatura científica lo hace a modo de sinónimo de "desarrollo instruccional" (instructional development), señalando los aspectos más técnicos de la enseñanza y del desarrollo de habilidades pedagógicas (definir objetivos y resultados de aprendizaje apropiados, rediseñar actividades, evaluación de los aprendizajes, alineamiento constructivo para favorecer el aprendizaje) (Lynn Taylor y Rege Colet, 2010: 142; Stes, MinLeliveld, Gijbels y Van Petegem, 2010).

No obstante, como señala Gibbs (2004; 2009), parece que estas estrategias centradas en la transformación de las prácticas docentes individuales en dirección de abajo arriba no son suficientes para una universidad inmersa en un mundo globalizado o para llevar a cabo un cambio de la universidad como un "todo". El concepto de "desarrollo docente" (faculty development de uso predominante en USA o academic development en Australia y Reino Unido) viene a resituar el anterior dándole un enfoque más holístico comprendiendo aspectos relativos al desarrollo profesional e instruccional pero también al desarrollo organizacional (Lynn Taylor y Rege Colet, 2010: 143). Hoy en día un programa de desarrollo docente debería operar desde una visión comprensiva (integrada), construirse bajo los parámetros del desarrollo profesional, el desarrollo instruccional, el desarrollo del liderazgo docente y el desarrollo organizativo (Wilkerson e Irby, 1998) y afectar a las conductas, concepciones y enfoques docentes sobre el aprendizaje de los estudiantes así como al aprendizaje mismo de los estudiantes y a la cultura organizativa (Gibbs y Coffey, 2004; Kirkpatrick, 1994; Prebble et al. 2004). Recientemente Lynn Taylor y Rege Colet (2010) señalan la ruptura entre el concepto de desarrollo docente y teorizan el concepto de "desarrollo educacional" como el más acorde con la realidad actual y que recoge todos los aspectos (instruccionales, profesionales, curriculares, académicos y organizativos) que permiten convertirse a las universidades en organizaciones que optimizan su capacidad de enseñar y aprender (Senge, 1996).

Teniendo en cuenta todo lo anterior, el programa ERAGIN (en vasco "influir") fue diseñado intencionalmente para que los docentes aprendieran a trabajar con metodologías activas en su plano individual pero de manera que los impulse a llevarlas a la práctica de forma compartida (cambio de cultura en sus entornos de equipo docente o de facultad) lo cual requiere del desarrollo de un liderazgo educativo distribuido (roles de formador, de difusor de experiencias, de coordinador de equipos docentes) y de una actuación "académica" docente (investigación de los procesos de enseñanza-aprendizaje hasta el nivel de la excelencia visible a la que hacíamos alusión). Por tanto, se trata de hacer emerger un rol docente académico y líder de un curriculum híbrido que funcione a modo de virus 
dentro del "tejido" universitario. Por tanto, hablaremos de ERAGIN como un programa de desarrollo docente en el sentido holístico que hemos indicado.

Igualmente hay que poner atención a la eficiencia de las modalidades y estrategias de desarrollo docente. La investigación señala que pese a que los cursos y seminarios de corta duración son los más comunes, las estrategias más efectivas están a menudo asociadas con intervenciones que exigen el aprendizaje activo y experiencial a lo largo del tiempo (Prebble et al. 2004; Steinert et al., 2006; Stes, Clement y Van Petegem, 2011; Southwell y Morgan, 2009), mientras que las de corta duración sirven apenas para propagar políticas institucionales o aprendizajes de carácter instrumental y puntual. Aspectos como el uso de aprendizaje experiencial, provisión de feed-back, relación efectiva entre pares e iguales, intervenciones bien diseñadas de acuerdo con los principios del aprendizaje y la enseñanza y el uso de diversos métodos educativos son especialmente relevantes para un efectivo desarrollo docente (Steinert et al. 2006).

Con estas premisas iniciales, el programa optó por una estrategia prolongada en el tiempo que creara condiciones para activar a los docentes desde sus necesidades prácticas y desde su conocimiento disciplinar y docente pero optando por estrategias que favorecieran el aprendizaje entre iguales. Adoptamos la mentoría colaborativa o comentoría, entendida como un proceso de transmisión horizontal de conocimiento, capital social y soporte psico-social que es percibido por quien participa en él como relevante para su trabajo y que conlleva una comunicación fluida, normalmente directa y sostenida en el tiempo entre una persona que se considera que tiene un mayor conocimiento relevante, sabiduría o experiencia (el mentor) y una persona que se percibe que tiene menos (el protegido) (Bozeman y Feeney, 2007: 731). Entendimos así mismo que la co-mentoría no podía ser mantenida en su tradicional acepción compensatoria y de inserción e inducción de noveles que es la más habitual en el ámbito universitario, para enfatizar en el aprendizaje entre todos los colegas que se comprometen con la enseñanza, el aprendizaje y el cuidado, para hacer el aprendizaje de los estudiantes lo más exitoso posible (Hargreaves y Fullan, 2000). No hay edades para ser mentor, lo importante es la experiencia previa que éste pueda aportar de cara a coadyuvar una determinada relación de co-mentoría en la que siempre se dan cita dos o más profesionales "iguales” que trabajan juntos para conseguir un objetivo. Trasladar esta idea a nuestro contexto puede significar en ocasiones que un profesor con menor trayectoria académica es más experto en metodología docente que otro que institucional y laboralmente está más consolidado. La co-mentoría se convierte así en un ejercicio sinergético que vincula a varios docentes en ejercicio que se desarrollan profesionalmente a la vez que empujan la eclosión de culturas organizativas que rompen el aislamiento y la jerarquía, permiten cruzar "fronteras" disciplinares, reconstruyen sus ideas sobre la enseñanza y cambian su práctica (Kochan y Trimble 2000; Pololi y Knight, 2005).

Teniendo en cuenta estas bases teóricas, se diseñó un programa de carácter voluntario y de larga duración (14 ETCS y 350 de trabajo acreditado) en el que se compaginan diferentes estrategias o modalidades de formación. Como se puede observar en la Tabla 1 las tres primeras modalidades se desarrollan a lo largo del primer semestre del año natural, mientras que las modalidades 5 y 6 se llevan a cabo ya en el aula y constituyen la ejecución práctica del diseño de la metodología activa. La co-mentoría es la estrategia que atraviesa de forma sostenida todo el proceso. Los mentores son profesores y profesoras que tienen una experiencia previa comprobada en la práctica de estas metodologías bien porque se han formado en otras universidades relevantes en el desarrollo de estas metodologías como MacMaster o Maastricht o bien porque ya han sido formados en el propio programa y han llevado a cabo de forma exitosa su trabajo. La selección de los mentores se realiza en base a la competencia que han demostrado en el desarrollo de la metodología y en base a la cercanía disciplinar respecto al grupo con el que van a colaborar. Este último punto es especialmente importante ya que son conocedores de las materias sobre las que van a aconsejar. $L$ a Tabla 1 pretende esbozar el significado de cada una de las modalidades así como su duración, intensidad y dinámicas derivadas.

TABLA 1. Diferentes fases y modalidades del programa ERAGIN

\begin{tabular}{|l|l|}
\hline Modalidades & Características \\
\hline $\begin{array}{l}\text { 1. Taller inicial. Intensivo } \\
\text { (enero) }\end{array}$ & $\begin{array}{l}\text { Taller con expertos para reflexionar colectivamente sobre las necesidades } \\
\text { profesionales. Ejemplificaciones de metodologías activas. Se resalta un nuevo rol } \\
\text { del profesor/a asociado a las metodologías activas }\end{array}$ \\
\hline $\begin{array}{l}\text { Modalidad: Presencial. Intensivo. 15-25 horas, en 3-4 días } \\
\text { 3 talleres, uno por metodología. }\end{array}$ \\
\hline $\begin{array}{l}\text { 2. Grupos de co- } \\
\text { mentoría (6 meses). }\end{array}$ & $\begin{array}{l}\text { Cada participante re-diseña al menos el 25\% del tiempo total de su propia } \\
\text { asignatura, según los criterios del MdC, el ABP o el ABPy. }\end{array}$ \\
\hline
\end{tabular}




\begin{tabular}{|c|c|}
\hline & $\begin{array}{l}\text { Cada participante, apoyado por su grupo de co-mentoría, enfrenta los } \\
\text { escenarios problemáticos desde su conocimiento disciplinar y práctico de la } \\
\text { docencia y re-diseña la asignatura. }\end{array}$ \\
\hline & $\begin{array}{l}\text { Modalidad: Extensiva, semi-presencial, flexible pero con hitos pre-fijados. } \\
\text { - } 4 \text { sesiones presenciales por grupo de co-mentoría (tres/cuatro participantes) } \\
\text { - Trabajo on-line (Moodle: foros; Skype: reuniones virtuales) } \\
\text { - Trabajo pautado a través de productos escritos. Contraste y valoración entre } \\
\text { pares y entre el mentor y cada participante. }\end{array}$ \\
\hline $\begin{array}{l}\text { 3. Taller para evaluar el } \\
\text { re-diseño. }\end{array}$ & $\begin{array}{l}\text { Taller donde se analiza con el mentor y el grupo el diseño. Se utiliza un check-list } \\
\text { para evaluar si la planificación de la metodología puede ser implementada con } \\
\text { garantías y puestas en común entre los participantes. } \\
\text { Modalidad: Presencial. Intensiva. } 5 \text { horas. } \\
3 \text { Talleres, uno por metodología }\end{array}$ \\
\hline \multirow[t]{2}{*}{$\begin{array}{l}\text { 4. Mentoría en la } \\
\text { implementación } \\
\text { diseño realizado. }\end{array}$} & $\begin{array}{l}\text { Puesta en práctica con el aula. Comunicación de vivencias personales en el } \\
\text { marco de la co-mentoría. Se aplican diferentes herramientas de evaluación del } \\
\text { aprendizaje logrado por los estudiantes. }\end{array}$ \\
\hline & $\begin{array}{l}\text { Modalidad: Extensiva, on-line (foros para comunicar los avances, dificultades). } \\
\text { Aulas abiertas: los participantes "invitan" a sus compañeros de co-mentoría a } \\
\text { asistir al aula para hacer observación directa y contraste. }\end{array}$ \\
\hline \multirow[t]{2}{*}{$\begin{array}{l}\text { 5. Validación final y } \\
\text { publicación del material } \\
\text { (Excelencia visible de la } \\
\text { enseñanza y el } \\
\text { aprendizaje) }\end{array}$} & $\begin{array}{l}\text { El participante, terminada la implantación práctica, realiza un análisis, introduce } \\
\text { modificaciones en su diseño, y presenta el trabajo final. } \\
\text { Los mentores y la dirección del programa evalúan y en su caso dan el visto bueno } \\
\text { para la publicación. }\end{array}$ \\
\hline & $\begin{array}{l}\text { Modalidad: Extensiva } \\
\text { publicación en el centro de recursos metodológicos de la UPV/EHU } \\
\text { (http://www.ehu.es/es/ikdbaliabideak) }\end{array}$ \\
\hline
\end{tabular}

El trabajo de los grupos de co-mentoría se ha desarrollado sobre la base de un guión realizado por la dirección del programa ERAGIN y consensuado con los mentores. El guión de los grupos de co-mentoría del primer semestre (modalidades 1,2 y 3 , tabla 1 ) se basa en el tratamiento que se puede dar a tres situaciones problemáticas frecuentes en la enseñanza:

a. ¿Cómo transformar el $25 \%$ del programa de mi materia en un diseño de enseñanza-aprendizaje activo coherente con las competencias a desarrollar? Es el primer problema práctico al que se enfrentan los participantes y habitualmente lleva consigo un ejercicio de integración de varios "temas" de la asignatura. Se trata de definir cuál será el escenario del problema, proyecto o caso, extraído de la realidad o práctica profesional, que origina la necesidad de desarrollar las competencias y los temas relacionados con el mismo. Concretar los resultados de aprendizaje, establecer un hilo conductor, y secuenciar y organizar los contenidos, de manera que los estudiantes los trabajen cuando son necesarios en el desarrollo del problema, y no de manera previa, son algunos de los aspectos a discutir en la co-mentoría.

b. ¿Qué estrategias docentes se deben seguir para fomentar y evaluar un aprendizaje coherente con la metodología? Se trata de proponer una posible secuencia de actividades que seguirán los estudiantes en la resolución del problema, proyecto o caso. Debe plantearse el tipo de actividad o tarea más idónea para el desarrollo de las competencias propuestas, siendo coherente además con la metodología. También se aborda cómo se realizará el seguimiento del aprendizaje logrado con las actividades, y cómo serán evaluadas, siguiendo un enfoque de evaluación formativa. Se deben definir criterios de evaluación claramente relacionados con los resultados de aprendizaje que se pretenden evaluar.

c. ¿Es el diseño realizado coherente con la metodología y su planificación puede ser implementada con garantías? Esta cuestión se aborda después de haber llevado a la práctica el diseño metodológico nuevo. La co-mentoría se centra en analizar globalmente las propuestas finales desarrolladas; comprobar su coherencia con el método y el enfoque cooperativo; contrastar las diversas opciones presentadas; valorar si los tipos de tareas elegidos, el seguimiento y evaluación del aprendizaje, y su planificación son coherentes y viables en su conjunto; y prever posibles dificultades en su implementación. 
Estas situaciones problemáticas que secuencian la co-mentoría no son meros foros de discusión sino que constituyen una parte del ciclo de trabajo con los productos escritos obligatorios que los participantes han de entregar a lo largo del proceso. Entendemos que los profesores participantes deben trabajar la reflexión de forma abierta y oral, pero también sistemática y escrita. Los productos escritos son la herramienta que nos permite mediar en esta revisión del pensamiento y de la acción docente y actuar de forma académica en el ámbito de la docencia (sistematización, rigurosidad y evaluación entre pares).

\section{Método}

La investigación presenta las características peculiares asociadas al desarrollo docente, es decir, se produce en un ámbito de práctica y es, por tanto, contextual y situada. En consecuencia, el acercamiento metodológico pasa por comprender cómo se han gestado y desarrollado estos procesos de manera local, convirtiéndose en una investigación orientada a la acción (applied/action-oriented research) diferente en su naturaleza a la investigación guiada por la teoría (theory driven research) (Kolmos, 2010:64). Como indican Steinert et al. (2006:519) la mayoría de los estudios que se han realizado en torno al desarrollo docente se han producido en y para un determinado contexto y en este sentido cada contexto es clave; no obstante, aunque los resultados de las investigaciones no son generalizables sí que lo pueden ser los principios que subyacen en ellas.

El programa ERAGIN ha sido analizado a través de un ciclo permanente de investigación-acción dentro del equipo responsable de formación (los autores del trabajo). Se ha puesto especial atención en el carácter social de la investigación/acción (Carr y Kemmis, 1986), es decir, ir más allá de la comprensión profunda de la práctica y buscar la transformación de los contextos en aras de mayor horizontalidad, participación y equidad. El trabajo de contraste interno inicial se ha ido abriendo a los grupos de mentores de cada una de las metodologías de manera que se ha construido un lenguaje común y una comprensión convergente y profunda de la práctica compartida.

\subsection{Objetivos de la investigación}

La investigación tiene como objetivo general producir evidencia empírica de forma longitudinal sobre la efectividad de esta estrategia de desarrollo docente. De forma más específica se pretende:

a) Analizar el cambio de las concepciones previas sobre enseñanza y aprendizaje de los docentes participantes.

b) Analizar los cambios que los participantes observan en su desempeño práctico en el aula.

c) Conocer la valoración que hacen los participantes de la co-mentoría como forma alternativa del trabajo docente, tanto en la fase de diseño como en la de implantación práctica de la metodología en el aula.

d) Valorar el nivel de activación de los participantes en el liderazgo educacional (emergencia de nuevos roles) y en la excelencia visible de la enseñanza y el aprendizaje (desarrollo organizativo/institucional), un año después de finalizar el programa.

En el gráfico 1 se presenta de forma gráfica la lógica que enlaza los tres niveles de impacto que pretenden medirse y evidenciarse a través de los objetivos de la investigación, o lo que es lo mismo, evidenciar la capacidad de impacto que tiene un programa de prolongado en el tiempo y basado la co-mentoría en los niveles individuales, colectivos e institucionales de la universidad. 
Gráfico 1. Papel de la co-mentoría en el tránsito de los niveles individuales a los niveles institucionales del aprendizaje

Impacto en el docente como individuo (privado, invisible)

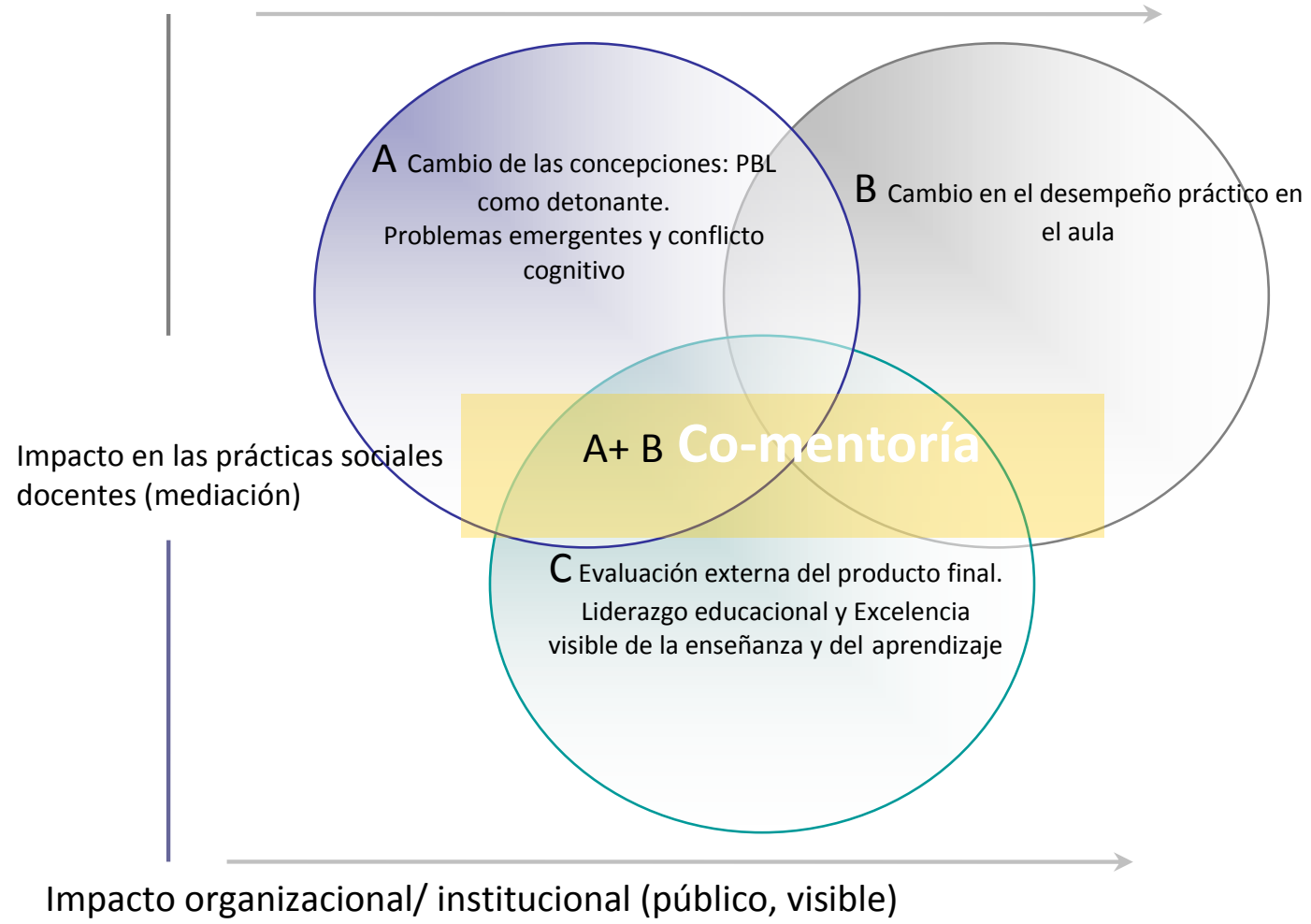

\subsection{Muestra}

En esta investigación para analizar los tres primeros objetivos hemos utilizado una muestra de 164 docentes, 74 de la primera edición del programa (2009-2010) y 90 de la segunda (2011-2012). Por áreas de conocimiento 18 son de Humanidades, 9 de Bellas Artes, 10 de Ciencias de la Salud, 31 de Ciencias Sociales, 31 de Ciencias Experimentales y 47 Tecnología.

El cuarto objetivo, en la medida de que pretende analizar el impacto organizacional e institucional, requiere que haya trascurrido un tiempo desde la finalización del programa. Por esta razón se ha optado por considerar como muestra sólo los 74 participantes de la primera promoción del programa, que finalizaron en junio de 2010.

\subsection{Instrumentos de recogida de información.}

La recogida de datos se ha realizado a través de:

a) Cuestionario preliminar de respuesta abierta para indagar las concepciones iniciales del profesorado participante sobre el proceso de E-A en su disciplina;

b) Los productos escritos de desarrollo de la propuesta de los participantes;

c) Cuestionario "valoración de la co-mentoría", realizada por cada participante al final de la fase de diseño de las propuestas y al final de la implementación en el aula de las propuestas diseñadas, y entrevista semiestructurada al $20 \%$ de los participantes;

d) Análisis del informe final de cada participante en relación con la mentoría y los resultados obtenidos tras la implementación;

e) Cuestionario sobre el impacto del programa a lo largo del tiempo (dos años después de la finalización de la primera promoción)

\subsection{Procedimiento de análisis de los resultados}


El análisis de los cuestionarios de respuesta abierta, los productos escritos, y los informes finales de implementación se ha llevado a cabo a través de tres estrategias (Kvale, 1996): 1) Codificar la frecuencia con que aparecían determinadas ideas o temas; b) Anotar patrones que pueden proceder de explicaciones causales o de determinadas ideas; c) Agrupar las principales unidades de contenido en categorías. Dos miembros del equipo examinaron independientemente los datos para realizar la primera codificación y generar patrones para cada categoría. En un segundo paso, las categorías iniciales fueron comparadas y reformuladas para indicar patrones específicos y categorías asociadas con diferentes aspectos del programa tales como: a) los objetivos de enseñanza del profesorado al comenzar el programa y las estrategias didácticas que utilizan; b) en qué medida la co-mentoría es entendida como mediación y ayuda en el proceso de cambio; c) calidad de los materiales didácticos basados en metodologías activas y de su implementación. En tercer lugar, con objeto de unificar criterios a la hora de asignar las respuestas a las diversas categorías, una muestra aleatoria del $20 \%$ de las asignaciones fue comparada por dos miembros del equipo, teniendo que llegar a un consenso en la asignación. Los criterios consensuados se aplicaron al resto de respuestas. En caso de desacuerdo, se procedía a un contraste final hasta llegar al consenso.

\section{Resultados}

\subsection{El "antes": ¿cómo comprenden los profesores la gestión del curriculum al comienzo del programa?}

La explotación y categorización de los 164 cuestionarios iniciales de respuesta abierta, dan como resultado una muy alta frecuencia de las categorías que encapsulan ideas típicas de un modelo de enseñanza basado en la transmisión de conocimiento disciplinar, lo cual es de esperar en una universidad con una tradición como la nuestra. Se indican a continuación las categorías establecidas y sus porcentajes:

I. Un $83 \%$ manifiesta ideas relativas a que su objetivo principal es que el estudiante aprenda los contenidos conceptuales más importantes de la asignatura.

II. Un 93\% manifiesta ideas que atribuyen al estudiante la responsabilidad exclusiva del aprendizaje.

III. Un 92\% no manifiesta en sus respuestas ninguna referencia a las concepciones alternativas de los estudiantes.

IV. Un 92\% manifiestan organizar el currículo de acuerdo con la organización jerárquica de conceptos y leyes de la propia disciplina, mientras que más del $80 \%$ de las respuestas atribuye a la enseñanza la función de transmitir contenidos conceptuales.

V. Un $8,5 \%$ esbozan escenarios y actividades para aplicar procedimientos, reflexionar y trabajar en grupo.

VI. Un 13\% manifiestan su intención de desarrollar competencias entre los estudiantes.

\subsection{Conflictos cognitivos ante el cambio en los modos de enseñar (impacto en las concepciones)}

Una vez que los participantes han recibido la formación del taller inicial y se ponen a desarrollar las tareas asociadas a los productos escritos, emergen tres grandes problemáticas que han debido ser tratadas, discutidas $y$ reconstruidas en cada grupo de co-mentoría:

I. Una tendencia, bastante recurrente entre los participantes, a creer que los estudiantes no pueden abordar Escenarios (problemas/proyectos/Casos) sin haber recibido previamente la "teoría". Una manifestación de esta resistencia es formulada así: "Entiendo que una vez explicada la teoría se utilice un Caso para que los estudiantes apliquen lo aprendido, pero en el programa la idea es que los estudiantes analicen el Caso antes de haber dado la teoría y no creo que sea posible".

II. Una dificultad para establecer un problema estructurante que aglutine varios temas y que se resuelva mediante actividades con los estudiantes. En el análisis del segundo producto escrito encontramos ejemplos estándar como los siguientes: "Encuentro muy difícil idear un proyecto que exija ir conociendo los contenidos del tema"; "No encuentro un Caso que pueda adaptarse al temario que he elegido. Puede que sirva como ejemplo una vez acabado el tema pero no entiendo cómo proponerlo desde el principio".

III. Dificultad para coordinar coherentemente las estrategias de aprendizaje activo con los objetivos y las competencias a lograr." Por ejemplo, un profesor indicaba en su tercer producto escrito: "Ya tengo el Problema estructurante y las actividades, pero ahora me encuentro con que tenía que haber pensado sobre la relación con las competencias que quiero enseñar en el tema y con los objetivos de aprendizaje que tengo que evaluar". Otro ejemplo estándar es el siguiente: "Al ir comentando cada actividad veo que tengo que considerar si el objetivo coincide con las competencias que he propuesto y sobre todo, qué actividades puedo seleccionar para evaluar los indicadores de aprendizaje".

El tratamiento de los problemas mencionados llevó a un consenso entre los participantes y mentores que se refleja en el feedback de los productos escritos y que podemos resumir de la siguiente forma: 
A. El escenario (proyecto/problema/caso) propuesto hace sentir a los estudiantes el interés y la necesidad de aprender nuevos conceptos, métodos y/o teorías. Así mismo, permite al profesor/a guiar al estudiante en ese proceso de aprendizaje. Por tanto, primero se crea la necesidad, y luego se diseñan las actividades para el aprendizaje.

B. El diseño de actividades no sigue la secuencia de primero informar de la teoría y luego aplicarla. Al contrario, un diseño en metodologías activas consiste en introducir un problema, cuyo análisis y resolución lleve a los estudiantes a la necesidad de aprender nuevos contenidos (teoría, procedimientos...) que, según el caso, requiere una búsqueda activa por parte del estudiante que es apoyada por la bibliografía, el profesor/a, Internet...

C. Las actividades para desarrollar el problema deben estar bien secuenciadas entre sí y responder a los indicadores de aprendizaje. Así mismo, la coherencia entre objetivos de aprendizaje, actividades y evaluación de los resultados de aprendizaje de los estudiantes deben aparecer explícitamente incluyendo los criterios de evaluación.

Los problemas comentados constituyeron importantes nudos de contenido, equiparables al concepto de zona de desarrollo próximo (Vygotsky, 1979), sobre los que los grupos de co-mentoría debieron pensar y debatir, con objeto de ir construyendo nuevos significados más acorde con las teorías científicas (Branda 2011) y con el Modo 2 de conocimiento que se pretende introducir en este curriculum híbrido. El grupo de co-mentoría es un catalizador de estas preocupaciones y de sus soluciones y se convierte en un espacio de aprendizaje social en el que los participantes sienten la necesidad de dar respuestas a problemas desafiantes, se activan para buscar soluciones y se prestan mutuamente soporte psico-social y conocimiento. Se trata sin duda de una situación de aprendizaje que guarda un gran isomorfismo con lo que posteriormente llevarán al aula con la metodología activa.

\subsection{Valoración de la co-mentoría durante la fase de diseño de propuestas.}

La valoración de la co-mentoría en esta primera fase alcanza valores medios muy satisfactorios (7,5 sobre 10 en el item "valoración global de la mentoría" y 7,24 en "Me ha enriquecido el debate con el mentor"). Se destaca como relevante la buena disposición de los mentores en hacer reflexionar al grupo, el saber orientar las dificultades y el aportar sugerencias valiosas con ejemplos de otras experiencias y bibliografía. Estos aspectos se confirman en las entrevistas que se tuvieron con el $20 \%$ de los participantes, con testimonios tales como: "los ejemplos aportados por mi mentora me han ayudado a 'visualizar' el diseño del material y las actividades. La reflexión en grupo excelente"; "se ha aportado información clarificadora como el documento del comentario de las actividades. La disposición de mi mentor muy buena".

Finalmente el documento de re-diseño inicial con que culmina esta primera fase del programa fue evaluado por el equipo de asesores y los mentores, pero ningún mentor podía evaluar a sus mentorizados. Esta evaluación es rigurosa e indica que el diseño cumple los requisitos para una práctica con garantías. Durante esta primera fase el $87 \%$ de los 164 participantes superaron esta fase satisfactoria o muy satisfactoriamente frente al 7,3\% de que se dio de baja voluntariamente y a un 5,4\% que no superó la evaluación.

\subsection{Co-mentoría e implementación práctica de la metodología activa (impacto en la práctica).}

La puesta en marcha de la metodología en las aulas universitarias requirió también atención y esfuerzo. Al igual que en la fase anterior, las dificultades fueron abordadas en los foros, las mentorías presenciales y se desarrollaron herramientas como los diseños de evaluación de las competencias adquiridas por los estudiantes (diseños estándar post-test o pre y post-test) y cuestionarios de evaluación de la actitud de los estudiantes hacia la metodología seguida.

Para evidenciar el impacto de la implementación utilizaremos como fuente de datos el cuestionario de valoración de la co-mentoría, y el informe que cada participante presenta al final del programa. Cabe destacar que:

Los resultados del cuestionario de valoración de la co-mentoría reflejan que el $50 \%$ del profesorado participante indica explícitamente que está muy satisfecho o y un $20 \%$ satisfecho con la co-mentoría. Un $20 \%$ realiza comentarios positivos y negativos, y un $10 \%$ no está satisfecho con la mentoría o con el programa en general.

En el informe que cada participante presenta al final del programa, el 70\% indica explícitamente que en el siguiente curso volverá a utilizar la nueva metodología y comenta los cambios de mejora que realizará. El otro $30 \%$ no hace comentarios explícitos sobre el tema, aunque la mitad indican las mejoras que introducirían para futuros cursos. 
El $40 \%$ de los informes finales hacen referencia a comentar con los colegas la experiencia realizada y en tratar de que vean sus ventajas.

En los informes finales una mayoría de profesores señalan que a la hora de llevar a la práctica la metodología las condiciones físicas y espaciales de las aulas, los grupos muy numerosos de estudiantes, el horario impuesto por el plan de estudios y la tradición del profesorado del Centro han sido elementos en ocasiones obstaculizadores. Sin embargo, estas cuestiones aparecen como marginales y más como incomodidades para realizar el trabajo que como aspectos importantes o decisivos. Los problemas sobre las posibles dificultades de la implementación se reflejan en las expectativas sobre su capacidad de innovar y sobre problemas que tradicionalmente han tenido al enseñar. A modo de ejemplo dos aportaciones:

“Cuando programé el 25\% del programa con metodología activa, sustituyendo la docencia magistral de los temas más importantes de la materia por trabajo en equipo, no estaba nada convencida de que los resultados de esta experiencia fuesen positivos. En mi fuero interno pensaba que el primer día que los estudiantes se descolocasen o mostrasen una actitud negativa ante el planteamiento de trabajo, abandonaba esta metodología y volvía a la docencia tradicional. La realidad ha sido diferente a mis nefastas previsiones y no ha habido ocasión de abandonar la experiencia" (no40, informe final).

"Puedo resumir el proceso en los siguientes puntos: a) Mucha mayor carga para la docente. Tremenda, diría yo. Mucha auto organización, dedicación e implicación de la profesora; b) Gran satisfacción por la marcha de la implementación, por la respuesta global del grupo y por los resultados. Necesidad de profundizar en algunos desajustes plasmados a lo largo del informe para futuras ocasiones; c) Necesidad de adecuar el sistema a otras asignaturas de mi área con mayor dificultad que la que ha sido objeto de implementación; d) Dificultades en el aula para trabajar en grupo. Necesidad de mesas móviles, no ancladas al suelo. Ha resultado incómodo; e) Muchas felicitaciones recibidas por el alumnado. Gratificante. Mayor aprendizaje humano y emocional. Más debate, reflexión y profundización. Un éxito y un descubrimiento para mí; f) Otro aspecto muy resaltado ha sido el importante incremento de la asistencia de los estudiantes a clase, así como su alto grado de implicación y compromiso mutuo que han alcanzado los estudiantes con sus equipos de trabajo" (no 81, informe final).

\subsection{El después: impactos organizativos e institucionales bajo un prisma de sostenibilidad.}

Los cambios que se han operado en nuestra universidad en el ámbito de la docencia son visibles, y obedecen a la alineación de este programa con la formulación del modelo educativo propio y a otro programa impulsado por la UPV/EHU de desarrollo de los grados en el que los equipos decanales y directivos asumen el liderazgo institucional en el ámbito docente (Fernández y Rekalde, 2011; Gibbs, 2004). No obstante, hemos pasado un cuestionario on-line a los docentes que participaron en la primera promoción de ERAGIN con objeto de ver el efecto que en el tiempo ha tenido el programa, en dos parámetros muy importantes desde el punto de vista de la intencionalidad inicial: el impacto en el liderazgo y en el ámbito de la excelencia visible de la E-A. De los 65 docentes que culminaron con éxito el programa han respondido a la encuesta el $63 \%$ con estos resultados:

I. Sostenibilidad en el tiempo y crecimiento intrínseco: El 92,6\% sigue manteniendo la metodología activa (MA) aprendida en ERAGIN (un total de 38 grupos de asignatura), y 24 de ellos afirma su intención de aplicarlo además a otras asignaturas a partir del curso 2012-2013.

II. Expansión externa: liderazgo docente distribuido inmediato. El 31,7\% de los participantes han implementado la MA "contagiando" a los otros profesores que imparten la asignatura, de manera que se han convertido en formadores de sus propios colegas en el uso de la metodología. El no neto que hemos cuantificado es de 30 profesores, aunque tenemos constancia de que son más.

III. Expansión externa: liderazgo docente distribuido mediato. El 36,5\% de los que han respondido a la encuesta se han involucrado en acciones de formación en cursos posteriores, ya sea como mentores del programa, ya dando pequeños talleres sobre estas temáticas en los centros que los han solicitado.

IV. Excelencia visible de la E-A. El 92,3\% del total de docentes que finalizaron el programa ha sido valorado positivamente para publicar su trabajo en IKD Baliabideak (UPV-EHU 2011 y 2012), centro de recursos para el aprendizaje de la UPV/EHU, publicados en código abierto. Además el $78 \%$ de los que han respondido a la encuesta han presentado posters, comunicaciones o han publicado artículos en revistas científicas sobre su experiencia de innovación metodológica.

\section{Discusión.}


El presente programa de desarrollo docente basado en la co-mentoría nos lleva a comprender que el cambio de la práctica docente (entendida como praxis) es un proceso continuo y duradero en el tiempo, que experimenta "idas y venidas" y requiere acompañamiento y maduración. Esto coincide con otras experiencias de formación de profesorado que indican la conveniencia de una formación prolongada en el tiempo (Feiman-Nemser, 2001; Stes et al. 2011). Es cierto que desde la investigación se han señalado también objeciones relacionadas con la dependencia del programa de formación del contexto académico (Steinert et al. 2006). Se indica que el profesorado no tiene tiempo, que su evaluación profesional está centrada en la investigación y que la docencia no es valorada. Sin embargo, la investigación también aporta evidencias de que cuando el programa de formación conecta con las necesidades docentes del profesorado, éste se implica profesional y emocionalmente en el proceso (Vázquez, Jiménez y Mellado 2010). El trabajo aquí expuesto presenta resultados en la misma dirección. Se ha tratado de diseñar una herramienta eficaz para responder a las necesidades del profesorado de la Universidad del País Vasco.

El trabajo y los datos aportados se limitan este caso, lo que evidentemente restringe la generalidad de las conclusiones. La estructura de la Universidad del País Vasco es similar a la de otras universidades del Estado Español y en este sentido se podría generalizar la experiencia formativa. Sin embargo el programa desarrollado se inserta en una estrategia de cambio más global y ha recibido un apoyo institucional que ha sido clave para su buen funcionamiento y que puede establecer diferencias con otras universidades. El apoyo institucional del equipo rectoral se puede concretar en dos aspectos importante, por un lado, ha habido recursos económicos para el programa pero también para la gratificación y formación de los mentores y, por otro, quizás lo más importante, la práctica innovadora de los docentes del programa es oficial a todos los efectos dentro de la docencia de la UPV-EHU. La experiencia innovadora no se ha tratado como un "experimento pedagógico" sino como docencia oficial alineada con un reto estratégico de la universidad.

En el marco institucional donde se desarrolla el programa, los datos obtenidos ponen de manifiesto que es posible llevar a cabo estrategias de desarrollo docente con efectos individuales y organizativos siempre y cuando se tengan en cuenta las orientaciones derivadas de la investigación científica en el ámbito, se creen equipos de formadores que sigan los procesos en un constante ejercicio de investigación-acción y se trate de construir el cambio desde las propias disciplinas y con un prisma integrado y global (lejos de adiestramientos exclusivamente didactistas y fragmentarios) dando valor a la experiencia previa de los docentes participantes. El hecho de que los mentores sean igualmente profesores y que todos los miembros de cada grupo de co-mentorías pertenezcan a áreas disciplinares cercanas o afines facilitan de forma sensible la construcción del Contenido Práctico de la Enseñanza en un clima de confianza y comunicación.

En el ámbito de la investigación en formación del profesorado universitario queda aún mucho camino por recorrer. Por un lado atender a un análisis más sistemático del impacto de estas metodologías en un curriculum híbrido; por otro definir estrategias que permitan medir el impacto en el aprendizaje de los estudiantes y por último seguir analizando con más profundidad el desarrollo de un apoyo explícito de la institución universitaria a un liderazgo docente y a la propagación de una excelencia visible de la docencia y del aprendizaje, condiciones necesarias para ser una auténtica universidad que aprende.

\section{Referencias}

Araujo U.F. \& Sastre G.S, (Eds) (2008). El aprendizaje basado en problemas. Una nueva perspectiva de la enseñanza en la universidad.Barcelona: Gedisa.

Armstrong, Elizabeth G. (1997). A hybrid model of problem-based learning. En D. BouD, \& G. Feletti (Eds). The challenge of problem based learning. (137-150). London: Kogan Page,.

Barnett, R. (2000). University knowledge in an age of supercomplexity. Higher Education, 40: 409-422.

Barrows, H.S. (1988) The tutorial process. Springfield, IL: Southerm Illinois University School of Medicine.

Bolivar, A. \& Caballero, K. (2008). Cómo hacer visible la excelencia en la enseñanza universitaria. Revista Iberoamericana de Educación, № 46/8, 1-10.

Boyer, E.L. (1990). Scholarship Reconsidered: Priorities of the Professoriate. Princeton, New Jersey: Princeton University Press, The Carnegie Foundation for the Advancement of Teaching.

Bozeman, B. \& Feeney, M.K. (2007). Toward a useful theory of mentoring: A conceptual analysis and critique. Administration \& Society, 39: 719-739.

Branda, L. (2011) El aprendizaje basado en problemas y la genuina realidad, diario de un tutor. Educación Médica 14(3), 151-159.

Bryan L.A. \& Atwater, M.M. (2002). Teacher beliefs and cultural models: A challenge for science teacher preparation programs. Science Education 86, 821-839.

Carr, W. \& Kemmis, S. (1986) Becoming Critical. Education, knowledge and action research, Lewes: Falmer. 
Farmer, E. A (2004). Faculty development for proble,-based learning Eur J Dent Educ, 8:59-66.

Feiman-Nemser, S. (2001). Helping novices learn to teach: Lessons from an exemplary support teacher. Journal of Teacher Education, 52(1), 17-30.

Fernández, I. \& Rekalde, I. (2011) Una universidad que aprende: innovación y cambio educativo en la UPV/EHU. Leioa: Servicio de Publicaciones.

Gaff, J.G. \& Simpson, R. (1994). Faculty development in United States. Innovative Higher Education, 18(3), 167-176.

Gibbons, M., Limoges, C., Nowotny, H. \& Schwartzman, S. (1994). The New Production of Knowledge: the dynamics of science and research in contemporary societies. Londres: Sage.

Gibbs, G. \& Coffey, M. (2004). The impact of training of university teachers on their teaching skills, their approach to teaching and the approach of learning of their students. Active Learning in Higher Education, 5 (1), 87-100.

Gibbs, G. (2004) Mejorar la enseñanza y el aprendizaje universitario mediante estrategias institucionales. Educar, 33, 11-30.

Gibbs, G. (2009) Developing students as learners -varied phenomena, varied contexts and a developmental trajectory for the whole endeavour. Journal of Learning Development in Higher Education, 1, 1-12.

Gosling, D. (2008) Educational Development in the United Kingdom. Londres: Report to the Heads of Educational Development Group (HEDG).

Hargreaves, A. \& Fullan, M. (2000). Mentoring in the New Millennium. Theory into Practice, 39 (1), 50-56.

Irby, D. (1996). Models of Faculty Development for PBL; Advances in Health Sciences Education 1:69-81.

Kirkpatrick, D.L. (1994) Evaluating training programs: the four levels. San Francisco, CA, Berret-Koehler Publishers.

Kolmos, A. (2010) Danish faculty development strategies. En A. Saroyan \& M. Frenay Building teaching capacities in Higher Education: A comprehensive International Model. (61-81), Sterling, Virginia: Stylus.

Kochan, F. \& Trimble, S. (2000) From Mentoring to Co-Mentoring: Establishing Collaborative Relationships. Theory into practice, 39 (1), 20-28.

Kvale, S. (1996). Interviews. An introduction to qualitative research interviewing. Thousand Oaks, CA: Sage Publications.

Lynn Taylor, K. \& Rege Colet, N. (2010) Making the sift from faculty devlopment to educational development. A conceptual framework grounded in practice. En A. Saroyan \& M. Frenay Building teaching capacities in Higher Education: A comprehensive International Model. (139-167), Sterling, Virginia: Stylus.

Morales, P. (2010). Investigación e innovación educativa. REICE, Vol. 8 (2). 48-73.

Pololi, L. \& Knight, S. (2005). Mentoring Faculty in Academic Medicine. A new paradigm? Journal of General Internal Medicine, 20 (9), 866-870.

Prebble, T., Hargraves, H., Leach, L., Naidoo, K., Suddaby, G. \& Zepke, N. Al. (2004) Impact of student support services and academic development programmes on student outcomes in undergraduate tertiary education: $A$ synthesis of the research. Wellington, New Zealand: Ministry of Education.

Rege Colet, N. (2010) Faculty development in Switzerland. En A. Saroyan \& M. Frenay Building teaching capacities in Higher Education: A comprehensive International Model.(43-60), Sterling, Virginia: Stylus.

Saroyan, A. \& Frenay, M. (2010) Building teaching capacities in Higher Education: A comprehensive International

Model. Sterling, Virginia: Stylus.

Schön, D.A. (1992). La formación de profesionales reflexivos. Madrid: Paidós.

Schulman, L. (1987) Knowledge and teaching: Foundations of the new reform. Harvard Educational Review 57 (1), 1 22.

Senge, P.M. (1996). La quinta disciplina. El arte y la práctica de la organización abierta al aprendizaje. Barcelona: Granica.

Steinert, Y.; Mann, K., Centeno, A., Dolmans, D., Spencer, J., Gelula, M. \& Prideaux, D. (2006). A systematic review of faculty development iniciatives designed to improve teaching effectiveness in medical education: BEME Guide No.8. Medical Teacher, vol.28, No.6, 497-526.

Stes, A., Min-Leliveld M., Gijbels, D. \& Van Petegem, P. (2010) The impact of instructional development in higher education: The state-of-the-art of the research. Educational Research Review 5, 25-49.

Stes A., Clement M. \& Van Petegem P. (2011) The effectiveness of faculty training porgramme: long-term and institutional impact. International Journal for Academic Development. Vol. 12, No. 2, 99-109.

Southwell, D. \& Morgan, W. (2009) Leadership and the impact of academic staff development and leadership development on student learning outcomes in higher education: a review of literature. ALTC. Queensland: Australia.

UPV/EHU (2011) y (2012) Recursos IKD Baliabideak. ISSN 2254-9153. Recuperado el 27-XII-2012: http://www.ehu.es/es/web/ikdbaliabideak/home.

UPV/EHU (2012). Plan estrategikoa 2012-2017. Recuperado el 27-XII-2012: http://www.ehu.es/p200content/es/contenidos/plan programa proyecto/20130205 plan estrategico 2017/es info/adjuntos/plan estra tegico2012 eusk.pdf. 
Vázquez, J.M., Jinénez, R. \& Mellado, V. (2010). Los obstáculos para el desarrollo profesional de una profesora de enseñanza secundaria en ciencias experimentales. Enseñanza de las Ciencias 28 (3), p. 417-432.

Vygotsky, L. S. (1979) El desarrollo de los procesos psicológicos superiores. Buenos Aires: Grijalbo.

Wilkerson L.A. \& Irby, D.M. (1998). Strategies for Improving Teaching Practices: A Comprehensive Approach to Faculty Development. Academic Medicine, Vol. 73 No. 4, 387-396. 